\title{
Las corporaciones hoteleras españolas: determinantes internos y externos de su desempeño
}

\author{
The Spanish hotel corporations: Internal and external performance drivers
}

\author{
Rubén Lado-Sestayo ${ }^{1}$, Milagros Vivel-Búa ${ }^{2 *}$ \\ ${ }^{1}$ Universidad de La Coruña, España \\ ${ }^{2}$ Universidad de Santiago de Compostela, España
}

Recibido el 15 de enero del 2017; aceptado el 27 de Agosto del 2018

Disponible en Internet el 10 de novembre de 2018

\section{Resumen}

Este trabajo realiza un estudio empírico de los determinantes del desempeño de los grupos hoteleros españoles. La muestra de análisis está formada por 1,019 establecimientos hoteleros afiliados a corporaciones hoteleras y el horizonte temporal es 2005-2011. Los resultados denotan que los factores característicos de la demanda de servicios hoteleros y de la estructura del mercado afectan significativamente al desempeño. Ello subraya la importancia de la gestión del punto turístico donde se localiza. Asimismo, también destaca, a nivel de hotel, el efecto obtenido para variables representativas de la cuota de mercado que éste tiene, su nivel de activo y su volumen de deuda. Por tanto, los gestores del hotel y del destino son responsables del desempeño hotelero.

Códigos JEL: M13, M21, L83.

Palabras clave: Desempeño; Grupo hotelero; España; Ubicación; Hotel.

\footnotetext{
*Autor para correspondencia.

Correo electrónico: mila.vivel@usc.es (M. Vivel-Búa)

La revisión por pares es responsabilidad de la Universidad Nacional Autónoma de México.
} 


\begin{abstract}
This paper analyses the performance's drivers of Spanish hotel groups in a sample of 1,019 hotels affiliated to hotel chains and time horizon is 2005-2011. The results suggest that demand and the market structure significantly affect the hotel performance. This highlights the importance of the management of the tourist destination where the hotels is located. Also, at the hotel level, results also confirm the effect of variables that are representative of the market share, the asset level and the indebtedness. Consequently, both hotel and tourist destination managers are responsible of hotel performance.
\end{abstract}

JEL codes: M13, M21, L83.

Keywords: Performance; Hotel group; Spain; Location; Hotel.

\title{
Introducción
}

La evolución positiva de la demanda de servicios hoteleros ha potenciado el papel de las corporaciones hoteleras en la oferta hotelera española en detrimento de los hoteles independientes. De hecho, según datos de Hotelmarket para el año 2015, la amplia mayoría de la oferta hotelera española está en manos de corporaciones hoteleras en un porcentaje próximo al $80 \%$. No obstante, este tipo de grandes empresas también se han visto afectadas por la reciente crisis económica iniciada en el año 2007, si bien su impacto no ha sido homogéneo. Mientras algunos grupos hoteleros han tenido que asumir la declaración de concursos de acreedores o acometer procesos de refinanciación de su pasivo, por ejemplo, Hoteles Foxá, el grupo Urvasco y High Tech Hotels, otros han potenciado sus planes de expansión internacional, especialmente, por Latinoamérica, como es el caso de Meliá Hoteles, NH hoteles y Room Mate.

Este cambio en la estructura general del sector tiene consecuencias sobre diferentes grupos de interés dado que, por norma general, los grupos hoteleros tienen una mayor dimensión y, en consecuencia, sus decisiones y actuaciones tienen efectos sobre una comunidad de agentes más amplia. En esta línea, cabe destacar que el turismo, donde el sector hotelero es su principal componente, representa un 10.2\% del PIB español en 2010 y se incrementó hasta el $11.1 \%$ en 2015, según datos publicados por el Instituto Nacional de Estadística.

Las consecuencias del protagonismo alcanzado por las corporaciones hoteleras en la oferta española alcanzan otros factores más particulares como, por ejemplo, la gestión de su desempeño. Ello es debido a que, generalmente, en este tipo de empresas, su modelo de gestión de activos y su posibilidad de generar y aprovechar sinergias que, por ejemplo, se traduzcan en economías de escala, tienen incidencia en su rentabilidad. Además, debe considerarse que la propia supervivencia del hotel se ve afectada por la propia gestión que estas grandes empresas realicen de la misma, la cual no está exenta de complejidad y requiere un estudio en profundidad de los factores que pueden influirla (Li y Sun, 2012). Así, en este trabajo el foco se centrará en analizar el desempeño empresarial, medido a través de la rentabilidad económica debido a que este indicador relaciona los resultados empresariales con la inversión, y es la medida más utilizada en estudios previos (Sainaghi et al., 2017; Lado-Sestayo et al., 2016a).

El entorno competitivo tampoco es ajeno al cambio estructural provocado por la mayor presencia de grupos hoteleros (Becerra et al., 2013; Sami y Mohamed, 2014). Ello puede relacionarse con la aparición de procesos de integración que pretenden mantener o potenciar la posición competitiva de establecimientos hoteleros en un mercado que, como el caso de 
España, tiene, actualmente, una oferta dominada por este tipo de empresas que generan un alto volumen de empleo (Vivel-Búa et al., 2016).

En definitiva, todo este escenario justifica la relevancia de focalizar un estudio en este tipo de empresas hoteleras. De este modo, el objetivo de este estudio empírico es analizar el desempeño, medido a través de la rentabilidad económica, de los hoteles integrados en corporaciones hoteleras dentro del mercado español y durante el período 2005-2011. En particular, se evalúan qué factores puede influir en este indicador económico, considerando tanto aquellos relacionados intrínsecamente con el establecimiento hotelero, como otros más bien vinculados con el punto turístico donde éste se localiza. Así, la consideración de estas dos dimensiones, hotel y localización, constituye una de las principales aportaciones de esta investigación, pues la mayoría de estudios previos se focalizan en variables a nivel de empresa (Ivanov y Zhechev, 2012). Al mismo tiempo, dado la importancia internacional que tiene España como mercado turístico, este país es foco de análisis destacado, con el fin de obtener resultados extrapolables a otros mercados. De acuerdo con la Organización Mundial del Turismo (OMT), cabe destacar que España se encuentra entre los cinco destinos internacionales más importantes tanto en llegadas de turistas como en ingresos generados por el turismo. Además, es el primer país a nivel mundial en competitividad turística según el ranking del Foro Económico Mundial para el año 2015.

El trabajo se organiza en cinco secciones. Después de este primer apartado introductorio, la segunda sección expone el marco teórico al que esta investigación se suscribe, mostrando la literatura relacionada y el planteamiento de hipótesis. A continuación, la tercera sección identifica la base de datos utilizada y explica el estudio empírico realizado. El trabajo concluye con una cuarta sección de conclusiones y, posteriormente, la recopilación de las referencias bibliográficas en la quinta sección.

\section{Marco teórico}

A pesar de la importancia que tienen las corporaciones hoteleras en la oferta de servicios turísticos, existen pocos estudios focalizados en evaluar los determinantes de su desempeño. No obstante, la literatura previa sí reconoce la existencia de múltiples diferencias entre los hoteles integrados en estos grupos empresariales y los hoteles independientes (Gursoy y Swanger, 2007; Botti et al., 2009). Por ejemplo, Sami y Mohamed (2014) encuentran que las cadenas hoteleras presentan mejores resultados respecto a otros tipos de empresas hoteleras, si bien también evidencian una relación negativa entre su tamaño y su desempeño. De hecho, centrándonos en la naturaleza de los determinantes del desempeño hotelero, debe destacarse que las características del hotel han constituido los principales factores analizados en la literatura previa (Ivanov y Zhechev, 2012).

Desde el punto de vista de los modelos de negocio, existen diferencias entre los hoteles independientes y aquellos integrados en una corporación hotelera (Yeung y Lau, 2005; Damonte et al., 1997). Ello puede relacionarse con las economías del conocimiento que obtienen los hoteles ya integrados en la corporación, e incluso del incremento del desempeño que se obtiene al incrementar la cuota de mercado fruto de la filiación (Yeap, 2011; Sinclair y Stabler, 1997; Zhao, 1994). A este respecto, estudios previos encuentran que la mayor cuota de mercado puede tener consecuencias de tipo estratégico, al aumentar la capacidad de la corporación hotelera de influir sobre el precio en las interrelaciones con sus competidores 
(Yeap, 2011). En consecuencia, la cuota de mercado será el primer factor considerado en este estudio planteándose la siguiente hipótesis:

H1: La cuota de mercado impacta positivamente en el desempeño.

Una de las variables analizadas como determinante del desempeño es el número de estrellas, el cual presenta una relación positiva con el precio y consecuentemente incrementa la rentabilidad (Lado-Sestayo et al., 2017; Becerra et al., 2013). Becerra et al (2013) encuentran que los hoteles afiliados a corporaciones hoteleras presentan mayor precio y menores descuentos, si bien estos autores afirman que esta diferenciación horizontal es menos efectiva que la diferenciación en el nivel de calidad (vertical). Consecuentemente, debido a que el entorno competitivo es exógeno, los hoteles deben diseñar estrategias para reducir la competencia e incrementar su desempeño, por ejemplo, potenciando su diferenciación (Graff, 2011). Así, el número de estrellas será el segundo factor considerado y de acuerdo con la literatura previa se plantea la siguiente hipótesis:

H2: La calidad del hotel impacta positivamente en el desempeño.

Una estrategia de diferenciación a nivel horizontal muy popular es la filiación de un hotel a corporaciones hoteleras. De acuerdo con estudios previos, ello puede provocar una reducción de la probabilidad de insolvencia y favorecer unas mayores economías de escala (Baum e Ingram, 1998; Sinclair y Stabler, 1997). Además, esta integración puede generar externalidades (Lee y Jang, 2015; Tsang y Yip 2009). De hecho, éstas pueden hacer referencia al hecho de compartir conocimientos, o a la concentración de empleados con mayor grado de especialización y nivel de productividad (Glaeser y Resseger, 2010; Halling y Marnburg, 2008; Desrochers, 2001). Al mismo tiempo, estas externalidades pueden crear un entorno favorable a compartir infraestructuras o capital social entre hoteles dentro de la misma corporación (Kalnins y Lafontaine, 2004). Sin embargo, debe considerarse que, de acuerdo con estudios previos recientes, este efecto positivo requiere incurrir en costes derivados de la menor eficiencia asociada al mayor tamaño (Sami y Mohamed, 2014). Asimismo, las corporaciones hoteleras presentan mayores costes fijos, los cuales, si bien suponen economías de escala en períodos de crecimiento, también pueden, en períodos de baja demanda, suponer mayores dificultades y comprometer la supervivencia del hotel (Lado-Sestayo et al., 2016b). En consecuencia, el tamaño del hotel, como proxy de la posible existencia de economías de escala, es el tercer factor considerado en este trabajo y, de acuerdo con Lee and Jang (2015), se plantea la siguiente hipótesis:

H3: Las economías de escala impactan positivamente en el desempeño.

Dentro de los factores del hotel determinantes de la rentabilidad, estudios previos han considerado un conjunto de variables de control. Así, el nivel de deuda debido a que puede ejercer presión sobre decisiones estratégicas de la compañía afecta al desempeño (Liu \& Hung, 2006; Vivel-Búa et al., 2018). Otra de las variables consideradas ha sido el nivel de liquidez, debido a que un déficit o exceso pueden tener consecuencias importantes sobre la gestión del hotel (Borde,1998; Jensen, 1986). Esta relación ha sido estudiada en numerosos estudios, si 
bien el signo de su efecto no es concluyente (Kim et al.; 2012; Chen, 2013). Por tanto, ambas variables, endeudamiento y liquidez, junto con un indicador de la capacidad de la empresa de atender sus pagos a corto plazos, medido a través del fondo de maniobra, será considerado en este estudio.

Los factores externos también pueden afectar significativamente al desempeño, de acuerdo con la literatura. De hecho, Sami y Mohamed (2014) encuentran un efecto notable de este tipo de variables sobre el desempeño y Sainaghi $(2011,2010)$ subraya la necesidad de considerar la ubicación geográfica del establecimiento hotelero en la evaluación de su desempeño en una muestra de hoteles independientes. De igual modo, Yang et al. (2015) muestra que es necesario incorporar características relacionadas con el destino donde se ubica el hotel para analizar su performance. Así, el nivel de demanda ha demostrado tener un impacto positivo en el desempeño (Lado-Sestayo et al., 2016; Lado-Sestayo et al., 2017). De este modo, el nivel de demanda será el cuarto factor considerado en este estudio a partir de la siguiente hipótesis.

H4: El nivel de demanda impacta positivamente en el desempeño.

Con el objetivo de reflejar la heterogeneidad en la demanda hotelera entre destinos turísticos, debido a que en España conviven destinos de sol y playa de alta estacionalidad con destinos urbanos, culturales y rurales, se ha considerado el nivel de estacionalidad como variable de estudio (Ridderstaat et al., 2014). De acuerdo con Vivel Búa et al. (2017), la estacionalidad tiene un impacto negativo en el desempeño debido a que el sector presenta costes fijos elevados. Por ello, en contextos de alta estacionalidad los gestores deben adaptar los recursos a las demandas del mercado (Karamustafa and Ulama, 2010). Ello es especialmente difícil en los hoteles independientes y, en consecuencia, los hoteles afiliados podrían presentar una ventaja al compartir infraestructuras. Además, la concentración temporal de la demanda puede tener como consecuencia un aumento de precios, el cual unido al mejor desarrollo en estos contextos de los hoteles afiliados por su capacidad de reducir recursos y la mejor gestión de ingresos tendría un efecto positivo sobre su desempeño (Vives et al., 2018), planteándose la siguiente hipótesis.

H5: Una mayor variabilidad en la demanda impacta positivamente en el desempeño.

Finalmente, el último factor a considerar es el entorno competitivo. Así, la existencia de un entorno competitivo concentrado, donde puedan llevarse a cabo prácticas colusorias podría incrementar la rentabilidad (Lee, 2015). De acuerdo con estos resultados, las corporaciones hoteleras compitiendo en múltiples mercados podrían presentar mayores incentivos a la colusión y, por tanto, una mejor performance (Mauri 2016). En consecuencia, la estructura de mercado será el quinto factor considerado en este estudio. En esta línea, los procesos de afiliación podrían contribuir a establecer barreras de entrada frente a nuevos competidores, lo que puede favorecer el incremento del nivel de precios y, consecuentemente, del desempeño de los hoteles afiliados (Lado-Sestayo et al., 2016a; Lee, 2015; Conlin y Kadiyali, 2006; Zhao, 1994; Suzuki, 2013).

De acuerdo con los postulados de la Estructura-Conducta-Resultado (SCP), la existencia de colusión entre las empresas instaladas justificaría la relación entre la concentración de mercado y el desempeño (Cowling y Waterson, 1976; Davies, 1999). Este paradigma establece 
un marco analítico a partir del cual los resultados empresariales dependen de la conducta de las empresas, la cual a su vez depende de la estructura del mercado. Así, la estructura del mercado caracterizada por el grado de concentración de la oferta, el grado de concentración de la demanda, el nivel de diferenciación del producto y las barreras de entrada determina la intensidad competitiva. En este marco las empresas y los consumidores deciden sus estrategias, lo que determina el resultado empresarial.

La Escuela de Chicago también plantea una relación positiva entre la concentración de mercado y el desempeño, aunque ésta se fundamenta en la eficiencia, la cual es mayor en las grandes empresas (Demsetz, 1973; Williamson, 1975, 1985). Dado que esta investigación utiliza una proxy de las economías de escala (tamaño de la empresa), es factible validar el planteamiento de la SCP en línea con estudios previos incluyendo como factor determinante la estructura de mercado (Lado-Sestayo et al., 2016a), debido a que el efecto del tamaño se recoge a través de la proxy de economías de escala, por lo que la hipótesis a contrastar es:

H6: El nivel de concentración de mercado impacta positivamente en el desempeño.

Las hipótesis planteadas permitirán una mejor comprensión de los determinantes del desempeño de los hoteles afiliados a cadenas hoteleras, contribuyendo a identificar las principales diferencias existentes entre éstos y los hoteles independientes.

\section{Análisis empírico}

\section{Datos}

La muestra de estudio se ha construido partir de las siguientes fuentes de información:

- $\quad$ Base de datos del Sistema de Análisis de Balances Ibéricos (SABI), para la obtención de la información económica y financiera del hotel.

- $\quad$ Base de datos de Alimarket, para la obtención de información relativa a características del hotel. Alimarket es una empresa de generación de contenidos sectoriales, que dispone de una base de datos de hoteles entre otros sectores.

- Encuesta de Ocupación Hotelera del Instituto Nacional de Estadística español, para identificar características de los puntos turísticos donde ubican los hoteles.

El período temporal analizado se corresponde con el intervalo 2005-2011. La elección de este horizonte de estudio es debido a, en primer lugar, que no existe información pública disponible sobre el punto turístico donde se localiza el hotel en años anteriores al 2005. En segundo lugar, la no disponibilidad de información económica-financiera completa posterior al 2011 en las bases de datos consultadas en el momento de realización de esta investigación. Por ejemplo, SABI puede presentar un lapso de hasta dos años en la incorporación de todos los datos de cada empresa en su base. A ello hay que añadir la posibilidad, como así fue para algunas de las corporaciones analizadas, de demoras propias causadas por la empresa en la publicación de su información. En general, ello supuso la búsqueda de un equilibrio entre la inclusión de datos completos para cada hotel y el número de hoteles que componen la muestra final objeto de estudio. En tercer y último lugar, este periodo de estudio es relevante, a efectos 
de investigación, porque es representativo de una etapa caracterizada por una intensificación en los procesos de afiliación a corporaciones hoteleras en España que ilustra la situación actual del sector.

\section{Definición de variables y planteamiento econométrico}

Respecto a la variable dependiente a analizar, dado el objeto de estudio, ésta es el desempeño de cada hotel integrado en el grupo hotelero. Se aproxima a través de su rentabilidad económica, esto es, el cociente entre su BAIT (Beneficio Antes de Intereses e Impuestos) y sus ingresos totales. Se utiliza este indicador debido a que no presenta variaciones derivadas de la estructura de capital de la empresa y, por tanto, permite evaluar su impacto sobre la performance.

En relación con las hipótesis planteadas para las variables a nivel de hotel, a continuación se detallan las variables que se utilizarán para cada una de ellas:

H1: La cuota de mercado de cada hotel se calcula como el porcentaje de ingresos en el punto turístico.

H2: La calidad del hotel se mide a través del número de estrellas que posee.

H3: Las economías de escala se aproximan a través del tamaño del hotel, calculado como el logaritmo neperiano de su volumen de activo en balance.

Como variables de control del hotel se han utilizado el volumen de endeudamiento, el número de empleados, el nivel de liquidez aproximado por el flujo de caja, y el fondo de maniobra.

En relación con las hipótesis planteadas para las variables del destino turística, a continuación se detallan las variables que se utilizarán para cada una de ellas:

H4: El volumen de demanda del hotel se mide como el número anual de visitantes, en millones de individuos, en cada punto turístico.

H5: El nivel de estacionalidad es la variable utilizada para medir la variabilidad de la demanda. Ésta se define como la varianza del nivel de ocupación medio mensual.

H6: Un índice de Herfindahl es utilizado para medir el nivel de concentración de mercado, en línea con estudios previos (Lado-Sestayo et al., 2016a, Pan, 2005; Davies, 1999). Este índice se calcula como la suma de las cuotas de mercado al cuadrado de los hoteles de cada destino turístico. Valores más alto suponen mayor concentración y por tanto menor nivel de competencia. 
La validación de las hipótesis de trabajo se realiza a través de la estimación del siguiente modelo:

$$
\text { Performance }_{i J t}=\mathrm{c}+\mathrm{T}_{\mathrm{t}}+X_{i t} \beta_{1}+X_{J t} \beta_{2}+\varepsilon_{i J t}
$$

Donde,

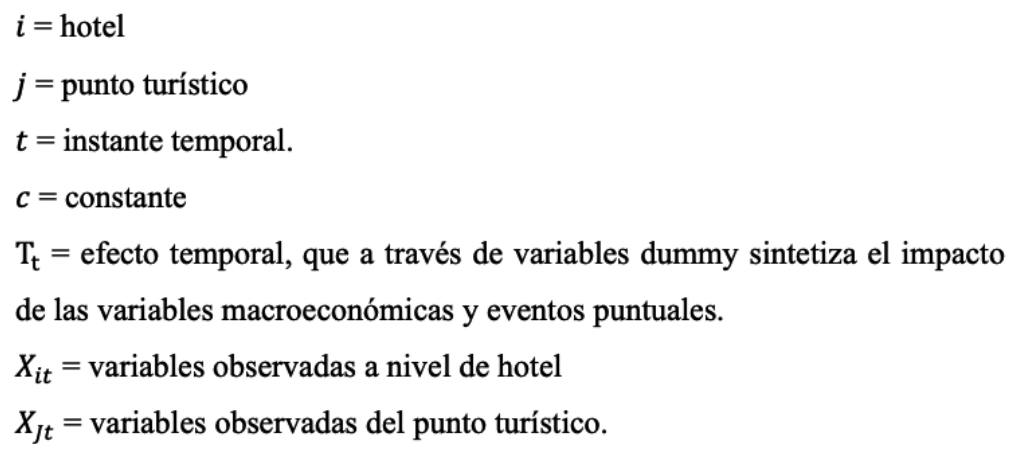

Las estimaciones se organizan en tres fases. Primero, se ha realizado una estimación por Mínimos Cuadrados Ordinarios (MCO). Posteriormente, se ha realizado la estimación por Mínimos Cuadrados Generalizados (MCG) a través de datos de panel con efectos aleatorios y efectos fijos, utilizando el test de Hausman para seleccionar el modelo más adecuado.

\section{Análisis estadístico - descriptivo}

La Tabla 1 identifica los estadísticos descriptivos de las variables que caracterizan el hotel y el punto turístico donde se ubica. Como resultados más destacados, podemos identificar que la dispersión de la cuota de mercado disminuye de un modo significativo en el período 20082010, lo cual puede ligarse a la crisis económica de estos años. Por su parte, el número de trabajadores se reduce notablemente desde el año 2008. Ello podría estar relacionado, junto con la evolución de la cuota de mercado, con la desaparición o quiebra de grupos hoteleros. El pasivo medio de los hoteles se incrementa a partir del año 2008 y hasta 2011. Al mismo tiempo, durante ese período de cuatro años, el flujo de caja y el fondo de maniobra caen drásticamente. Nuevamente, estos datos podrían estar explicar la quiebra de algunos grupos hoteleros, debido a las mayores dificultades para la devolución de un pasivo mayor en un contexto de menor liquidez.

A nivel de punto turístico, podemos observar que el número de visitantes ha crecido durante el período 2005-2011, con un descenso puntual en los años 2008 y 2009. Por tanto, por una parte, tenemos un incremento o mantenimiento del nivel de demanda, pero por otra, tal y como se indicó anteriormente, tenemos una contracción del flujo de caja en los hoteles. Quizás la caída en el nivel de concentración de mercado podría justificar que la evolución positiva de la demanda no se traslade directamente a la tesorería. Finalmente, cabe destacar que la estacionalidad cae durante el 2005-2007 pero se incrementa a partir del 2008 de forma notable. 


\section{Análisis de resultados}

La Tabla 2 presenta los resultados obtenidos a través de las estimaciones econométricas realizadas. En particular, el modelo 1 se refiere a la estimación por MCO, el modelo 2 a la estimación de datos de panel con efectos aleatorios, y el modelo 3 es la estimación con efectos fijos. De acuerdo con los estadísticos calculados para validar dichas estimaciones, podemos concluir que el modelo con efectos fijos es preferible al resto de estimaciones (modelo 3). Ello se debe a que el Test $\mathrm{F}$ de efectos fijos confirma que estos presentan significatividad estadística, al igual que el LM test de efectos aleatorios confirma la significatividad de los efectos aleatorios. Por tanto, ambos modelos (efectos fijos y efectos aleatorios) son preferibles al modelo estimado por MCO. Con el objetivo se seleccionar entre el modelo de efectos fijos y el modelo de efectos aleatorios se ha realizado el test de Hausman. Sus resultados apuntan a que el modelo de efectos fijos es preferible frente al modelo de efectos aleatorios.

Los resultados muestran que la demanda y la estructura de mercado son variables relevantes que influyen en la performance de los grupos hoteleros españoles. Por tanto, ello subraya la relevancia de las características del punto turístico cuando se evalúan la performance hotelera.

Si nos focalizamos en las características del hotel, las estimaciones permiten confirmar el efecto de la cuota de mercado y las economías de escala. Respecto a la primera, este resultado confirma que la cuota de mercado no necesariamente tiene que incrementarse sacrificando el margen de beneficios. En relación con las economías de escala, éstas pueden ser aprovechadas individualmente por cada establecimiento hotelero integrado en un grupo hotelero.

No se ha encontrado evidencia para la calidad del hotel, medida a través del número de estrellas que posee. Quizás ello es debido a que la calidad no sólo influye sobre el precio sino también sobre los costes. Ello podría suponer que son otros factores al margen de la calidad considerada únicamente, quienes pueden manifestar una incidencia sobre la performance hotelera.

Centrándonos en las variables de control, existe un efecto negativo del volumen de deuda sobre la performance. Ello puede relacionarse con la influencia que puede tener el endeudamiento sobre la decisiones estratégicas y tácticas vinculadas con el precio, las cuales podrían estar orientadas a la generación de flujo de caja más que una mejora de la performance.

Por su parte, el empleo tiene una relación negativa con la performance hotelera. Ello podría ser justificado por los costes asociados o incluso con la eficiencia marginal asociada al incremento de personal.

En relación al flujo de caja, este tiene un impacto positivo sobre la performance de los hoteles integrados en las corporaciones hoteleras.

Finalmente, se ha utilizado el método propuesto por Billor et al. (2000) para detectar la posible existencia de valores atípicos con incidencia en los parámetros estimados. Los resultados confirman la ausencia de valores atípicos. 


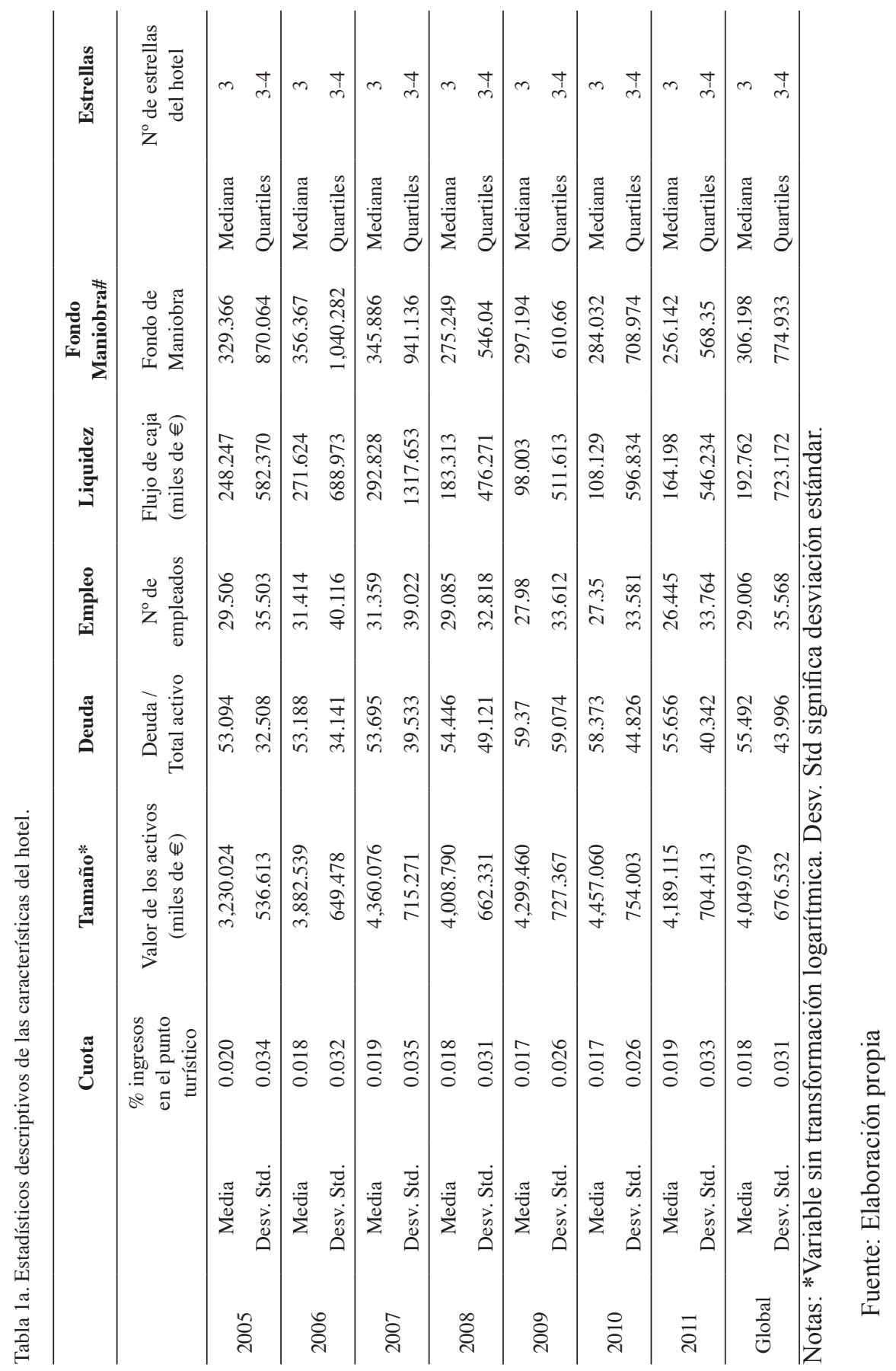


Tabla 1b. Estadísticos descriptivos de las características del destino turístico.

\begin{tabular}{lllll} 
& & Visitantes & Concentración* & Estacionalidad \\
\hline \multirow{2}{*}{2005} & Media & 1.057 & Índice de Herfindahl & Varianza de la ocupación mensual \\
& Desv. Std. & 1.443 & 0.033 & 0.229 \\
\hline \multirow{2}{*}{2006} & Media & 1.263 & 0.032 & 0.103 \\
& Desv. Std. & 1.724 & 0.033 & 0.21 \\
& Media & 1.342 & 0.034 & 0.096 \\
\hline \multirow{2}{*}{2008} & Mesv. Std. & 1.879 & 0.032 & 0.209 \\
& Media & 1.336 & 0.033 & 0.091 \\
\hline \multirow{2}{*}{2009} & Desv. Std. & 1.932 & 0.032 & 0.228 \\
& Media & 1.299 & 0.031 & 0.096 \\
\hline \multirow{2}{*}{2010} & Desv. Std. & 1.926 & 0.029 & 0.232 \\
& Media & 1.458 & 0.023 & 0.101 \\
\hline \multirow{2}{*}{2011} & Desv. Std. & 2.217 & 0.030 & 0.255 \\
& Media & 1.508 & 0.024 & 0.099 \\
\hline \multirow{2}{*}{ Global } & Desv. Std. & 2.292 & 0.036 & 0.257 \\
& Media & 1.323 & 0.031 & 0.092 \\
\hline & Desv. Std. & 1.938 & 0.032 & 0.232 \\
\hline
\end{tabular}

Notas: *Variable sin transformación logarítmica. Desv. Std significa desviación estándar.

Fuente: Elaboración propia

Tabla 2. Estimaciones econométricas.

\begin{tabular}{llll}
\hline & MODELO 1 & MODELO 2 & MODELO 3 \\
\hline & MCO & Efectos aleatorios & Efectos fijos \\
\hline Coef. & Coef. & Coef. \\
\hline Cisitantes & $0.362^{* * *}$ & $0.564 * * *$ & $1.414 * * *$ \\
Concentración & $(0.138)$ & $(0.122)$ & $(0.589)$ \\
& $0.317 * * *$ & $0.540^{* * *}$ & $0.800 * * *$ \\
Estacionalidad & $(0.030)$ & $(0.092)$ & $(0.093)$ \\
& 2.642 & 2.903 & 0.416 \\
Cuota & $(2.597)$ & $(4.031)$ & $(4.861)$ \\
& $17.335 * * *$ & $33.284 * * *$ & $58.980 * * *$ \\
Tamaño & $(6.592)$ & $(10.377)$ & $(21.788)$ \\
& $1.001 * * *$ & $3.715 * * *$ & $6.105 * * *$ \\
Estrellas & $(0.223)$ & $(0.431)$ & $(0.663)$ \\
& 0.039 & -0.688 & -0.879 \\
Deuda & $(0.265)$ & $(0.701)$ & $(0.821)$ \\
& $-0.201 * * *$ & $-0.217 * * *$ & $-0.207 * * *$
\end{tabular}




\begin{tabular}{|c|c|c|c|}
\hline & $(0.005)$ & $(0.006)$ & $(0.007)$ \\
\hline \multirow[t]{2}{*}{ Empleo } & $-0.042 * * *$ & $-0.077 * * *$ & $-0.084 * * *$ \\
\hline & $(0.008)$ & $(0.013)$ & $(0.017)$ \\
\hline \multirow[t]{2}{*}{ Liquidez } & $0.004 * * *$ & $0.004 * * *$ & $0.004 * * *$ \\
\hline & $(0.000)$ & $(0.000)$ & $(0.000)$ \\
\hline \multirow[t]{2}{*}{ Fondo de maniobra } & 0.000 & -0.000 & -0.000 \\
\hline & $(0.000)$ & $(0.000)$ & $(0.000)$ \\
\hline \multirow[t]{2}{*}{2006} & 0.493 & 0.335 & 0.037 \\
\hline & $(0.794)$ & $(0.548)$ & $(0.570)$ \\
\hline \multirow[t]{2}{*}{2007} & 0.298 & 0.554 & 0.322 \\
\hline & $(0.802)$ & $(0.556)$ & $(0.583)$ \\
\hline \multirow[t]{2}{*}{2008} & $-2.149 * * *$ & $-2.187 * * *$ & $-2.474 * * *$ \\
\hline & $(0.791)$ & $(0.548)$ & $(0.567)$ \\
\hline \multirow[t]{2}{*}{2009} & $-4.517 * * *$ & $-4.418 * * *$ & $-4.712 * * *$ \\
\hline & $(0.782)$ & $(0.546)$ & $(0.561)$ \\
\hline \multirow[t]{2}{*}{2010} & $-3.654 * * *$ & $-4.065 * * *$ & $-4.563^{* * *}$ \\
\hline & $(0.788)$ & $(0.568)$ & $(0.597)$ \\
\hline \multirow[t]{2}{*}{2011} & $-4.363^{* * *}$ & $-5.115 * * *$ & $-5.918 * * *$ \\
\hline & $(0.819)$ & $(0.603)$ & $(0.655)$ \\
\hline \multirow[t]{2}{*}{ Constante } & 3.387 & $-12.095 * * *$ & $-30.402 * * *$ \\
\hline & $(2.186)$ & $(4.075)$ & $(5.825)$ \\
\hline \multirow[t]{2}{*}{ Obs, (grupos) } & 5560 & $5560\left(\begin{array}{ll}1 & 019\end{array}\right)$ & $5560\left(\begin{array}{ll}1 & 019\end{array}\right)$ \\
\hline & 0.304 & 0.290 & 0.256 \\
\hline Test F (todos) & $151.44 * * *$ & & $103.6^{* * *}$ \\
\hline Wald ( & & $1.967 * * *$ & - \\
\hline LM test (Breushc-Pagan) & & $877.66^{* * *}$ & - \\
\hline Test F (Efectos Fijos) & - & - & $7.516^{* * *}$ \\
\hline Hausman () & & $52.90 * * *$ & \\
\hline VIF & 1.60 & & \\
\hline
\end{tabular}

Notas: Coef. representan los coeficientes con los errores estándar heterocedásticamente robustos entre paréntesis debajo. VIF es la media del factor de inflación de la varianza, que se utiliza como un indicador de multicolinealidad, obteniéndose un valor adecuado dado que presenta un valor inferior a dos. $* * *, * * * *$ significativo al $1 \%, 5 \%$ y $10 \%$ respectivamente.

Fuente: Elaboración propia

\section{Conclusiones}

En este trabajo se ha estudiado los determinantes de la performance de 1,019 hoteles afiliados a corporaciones hoteleras en España en el período 2005-2011. Para ello, se han considerado tanto variables que caracterizan de forma individual cada hotel, como también variables que 
caracterizan el punto turístico español donde éste se localiza. La incorporación de ambas dimensiones, esto es, hotel y punto turístico, supone una aportación de este trabajo ante el bajo número de estudios que incluyen variables externas al hotel en el estudio de su performance. Asimismo, la mayoría de estudios previos se han centrado en hoteles independientes a pesar de que se han encontrado diferencias notables entre estos y los hoteles afiliados a corporaciones hoteleras.

Los resultados obtenidos han contribuido a apoyar las hipótesis relativas al efecto positivo de la cuota de mercado, las economías de escala, el nivel de demanda y la concentración de mercado sobre el desempeño. Ello implica que los hoteles con una gran presencia en el mercado y un mayor tamaño tienen un mejor desempeño en línea con lo obtenido en estudios previos. Por tanto, aquellas actuaciones orientadas a la mejora de la cuota de mercado constituyen una vía para la mejora de los resultados. Este resultado confirma el impacto estratégico de la cuota de mercado sobre los competidores destacado en estudios previos. Asimismo, en lo relativo al impacto positivo del tamaño, se confirma la existencia de economías de escala, lo cual está en línea con la existencia de externalidades positivas evidenciadas en la literatura previa, justificando la mayor presencia de corporaciones hoteleras en el mercado en el horizonte de estudio considerado. Ello podría explicarse por el hecho de que los hoteles afiliados comparten costes de publicidad y gestión, además de presentar un mayor poder negociador frente a comercializadores y particularmente frente a las agencias de viajes online, que concentran gran parte de las oferta hotelera.

En relación a las hipótesis que no ha sido posible verificar, no se ha obtenido evidencia que apoye la existencia de un efecto positivo del nivel de calidad ni de la variabilidad de la demanda sobre el desempeño. Ello podría deberse a la diferenciación horizontal que presenta la afiliación, que podría compensar la ausencia de diferenciación en calidad vía un mayor número de estrellas. la diferenciación vertical (mayor número de estrellas) supone unos costes en línea con los mayores ingresos que genera. En síntesis, la divergencia en este resultado con investigaciones precedentes que analizan hoteles independientes podría encontrarse en el hecho de que el nivel de calidad es un indicador que favorece una mayor demanda en los hoteles independientes, si bien la imagen de la corporación hotelera actúa como indicador en los hoteles afiliados. En relación a la ausencia del efecto de la estacionalidad, Los resultados apuntan a que la mejor posición de los hoteles afiliados para adaptar los recursos a las demandas del mercado de acuerdo con la literatura previa, no es suficiente para compensar el efecto de los costes fijos.

En relación a las variables de control utilizadas, los resultados apuntan a el nivel de endeudamiento afecta negativamente al desempeño, mientras que la liquidez contribuye a que este aumente. Ante la falta de estudios previos que desde un punto de vista teórico soporten esta relación en el sector, estos resultados apuntan a la necesidad de profundizar en el impacto de la gestión financiera del hotel, dado que esta podría presentar un papel importante para la generación de resultados positivos. Entre otros aspectos, ello podría explicarse por la estructura de costes en el sector, muy dependiente de costes fijos y por el hecho de que el producto ofrecido sea perecedero.

Los resultados ponen de manifiesto que el análisis del desempeño en las corporaciones hoteleras debe considerar variables del hotel y del destino turístico y que, consecuentemente, los modelos planteados para hoteles independientes en la literatura previa pueden no ser válidos para hoteles afiliados. Además, estos resultados presentan áreas de interés común para gestores 
de hoteles y destinos turísticos, por ejemplo, para el fomento de la demanda del destino turístico. Sin embargo, también señalan que ante otro tipo de actuaciones, por ejemplo encaminadas a reducir la estacionalidad, los hoteles afiliados podrían no tener incentivos a la colaboración con los gestores del destino.

El trabajo ha tenido alguna limitación para su desarrollo. A pesar del esfuerzo realizado para la obtención de información completa sobre las corporaciones hoteleras a analizar, ello estuvo limitado por la disponibilidad de datos, condicionando y limitando el período de estudio. Asimismo, no fue posible disponer de información sobre el punto turístico en período anteriores a 2005.

\section{Referencias}

Baum, j. A., e ingram, p. (1998). Survival-enhancing learning in the Manhattan hotel industry, 1898-1980. Management Science, 44(7), 996-1016. https://doi.org/10.1287/mnsc.44.7.996

Becerra, M.; Santaló, J. \& Silva, R. (2013). Being better vs being different: differentiation, competition, and pricing strategies in the Spanish hotel industry. Tourism Management, 34, 71-79. https://doi.org/10.1016/j.tourman.2012.03.014

Borodako, K. (2011). Cooperation of small and medium-sized tourism enterprises with tourism stakeholders in the Maopolska region - top management perspective approach. Tourism \& Management Studies, 7 (1), 24-32.

Borde, S. F. (1998). Risk diversity across restaurants: An empirical analysis. Cornell Hotel and Restaurant Administration Quarterly, 39(2), 64-69. https://doi.org/10.1016/s0010-8804(98)80013-x

Botti, L., Briec, W., \& Cliquet, G. (2009). Plural forms versus franchise and company-owned systems: A DEA approach of hotel chain performance. Omega, 37(3), 566-578. https://doi.org/10.1016/j.omega.2007.12.002

Bresciani, S., Thrassou, A. \& Vrontis, D. (2015). Determinants of performance in the hotel industry - an empirical analysis of Italy. Global Business and Economics Review, 17(1), 19-34. https://doi.org/10.1504/gber.2015.066531

Chen, M. H. (2013). Risk determinants of China's hotel industry. Tourism Economics, 19(1), 77-99. https://doi. org/10.5367/te.2013.0183

Conlin, M., \& Kadiyali, V. (2006). Entry-deterring capacity in the Texas lodging industry. Journal of Economics \& Management Strategy, 15(1), 167-185. https://doi.org/10.1111/j.1530-9134.2006.00096.x

Cowling, K., \& Waterson, M. (1976). Price-cost margins and market structure. Economica, 43(171), 267-274. https:// doi.org/10.2307/2553125

Damonte, L.T., Rompf, P., Domke, D. \& Bahl, R. (1997). Brand affiliation and property size effects on measures of performance in lodging properties. Hospitality Research Journal, 20 (3), 1-16.

Davies, B. (1999). Industrial organization the UK hotel sector. Annals of tourism research, 26(2), 294-311. https://doi. org/10.1016/s0160-7383(98)00096-6

Demsetz, H. (1973). Industry structure, market rivalry, and public policy. The Journal of Law and Economics, 16(1), 1-9. https://doi.org/10.1086/466752

Desrochers, P. (2001). Geographical proximity and the transmission of tacit knowledge. Review of Austrian Economics, 14, 25-46. https://doi.org/10.1023/a:1007803520748

Enz, C.A. (2010). Hospitality Strategic Management: Concepts and Cases. New Jersey: John Wiley \& Sons.

Glaeser, E. L., \& Resseger, M.G. (2010). The complementarity between cities and skills. Journal of Regional Science, 50, 221-244. https://doi.org/10.1111/j.1467-9787.2009.00635.x

Graf, N. S. (2011). Market structure and demand-side substitutability of chained urban hotel segments. International Journal of Hospitality Management, 30(1), 82-90. https://doi.org/10.1016/j.ijhm.2010.03.011

Gursoy, D., \& Swanger, N. (2007). Performance-enhancing internal strategic factors and competencies: impacts on financial success. International Journal of Hospitality Management, 26(1), 213-227. https://doi.org/10.1016/j. ijhm.2006.01.004 
Hallin, C.A., \& Marnburg, E. (2008). Knowledge management in the hospitality industry: A review of empirical research. Tourism Management, 29 (2), 366-381. https://doi.org/10.1016/j.tourman.2007.02.019

Hostelmarket (2015). La restauración rápida busca alejarse de las prisas. Publicaciones Alimarket, 179 (Marzo).

Ivanov, S., \& Zhechev, V. (2012). Hotel revenue management-a critical literature review. Tourism : An International Interdisciplinary Journal, 60(2), 175-197. https://doi.org/10.2139/ssrn.1977467

Jensen, M. C. (1986). Agency costs of free cash flow, corporate finance, and takeovers. The American economic review, 76(2), 323-329. https://doi.org/10.1017/cbo9780511609435.005

Kalnins, A., \& Lafontaine, F. (2004). Multi-unit ownership in franchising: evidence from the fast-food industry in Texas. The RAND Journal of Economics, 35 (4), 747-761. https://doi.org/10.2307/1593771

Karamustafa, K., \& Ulama, S. (2010). Measuring the seasonality in tourism with the comparison of different methods. EuroMed Journal of Business, 5(2), 191-214. https://doi.org/10.1108/14502191011065509

Kim, H., Kim, J., \& Gu, Z. (2012). An examination of US hotel firms' risk features and their determinants of systematic risk. International Journal of Tourism Research, 14(1), 28-39. https://doi.org/10.1002/jtr.828

Lado-Sestayo, R., Otero-González, L., Vivel-Búa, M., \& Martorell-Cunill, O. (2016a). Impact of location on profitability in the Spanish hotel sector. Tourism Management, 52, 405-415. https://doi.org/10.1016/j.tourman.2015.07.011

Lado-Sestayo, R., Vivel-Búa, M., \& Otero-González, L. (2016b). Survival in the lodging sector: An analysis at the firm and location levels. International Journal of Hospitality Management, 59, 19-30. https://doi.org/10.1016/j. ijhm.2016.08.005

Lado-Sestayo, R., Vivel-Búa, M. \& Otero-González, L. (2017). Determinants of TRevPAR: hotel, management and tourist destination. International Journal of Contemporary Hospitality Management 29, (12), 3138-3156. https:// doi.org/10.1108/ijchm-03-2016-0151

Lee, S. K., \& Jang, S. (2015). Conditional agglomeration externalities in lodging markets. Journal of Hospitality \& Tourism Research, 39(4), 540-559. https://doi.org/10.1177/1096348013491605

Lee, S.K. \& Jang, S.C.S. (2012). Premium or discount in hotel room rates? The dual effects of a central downtown location. Cornell Hospitality Quarterly, 53 (2), 165-173. https://doi.org/10.1177/1938965512441056

Lee, S.L. (2015). Quality differentiation and conditional spatial price competition among hotels. Tourism Management 46, 114-122. https://doi.org/10.1016/j.tourman.2014.06.019

Li, H. \& Sun, J. (2012). Forescating business failure: the use of nearest-neighbour support vectors and correcting imbalanced samples - Evidence from the Chinese hotel industry. Tourism Management, 33 (1), 622-634. https://doi. org/10.1016/j.tourman.2011.07.004

Liu, Y. C., \& Hung, J. H. (2006). Services and the long-term profitability in Taiwan's banks. Global Finance Journal, 17(2), 177-191. https://doi.org/10.1016/j.gfj.2006.03.001

Mauri, A. G. (2016). Pricing and revenue management in hotel chains. The Routledge Handbook of Hotel Chain Management, pp. 262.

Ridderstaat, J., Oduber, M., Croes, R., Nijkamp, P., \& Martens, P. (2014). Impacts of seasonal patterns of climate on recurrent fluctuations in tourism demand: Evidence from Aruba. Tourism Management, 41, 245-256. https://doi. org/10.1016/j.tourman.2013.09.005

Sainaghi, R. (2010). Hotel performance: state of the art. International Journal of Contemporary Hospitality Management, 22(7), 920-952. https://doi.org/10.1108/09596111011066617

Sainaghi, R. (2011). RevPAR determinants of individual hotels: Evidences from Milan. International Journal of Contemporary Hospitality Management 23 (3), 297 - 311. https://doi.org/10.1108/09596111111122497

Sainaghi, R., Phillips, P. \& Zavarrone, E. (2017). Performance measurement in tourism firms: A content analytical meta-approach. Tourism Management 59: 36-56. https://doi.org/10.1016/j.tourman.2016.07.002

Sami, B. A., \& Mohamed, G. (2014). Determinants of tourism hotel profitability in Tunisia. Tourism and Hospitality Research, 14(4), 163-175. https://doi.org/10.1177/1467358414543970

Sinclair, M.T. \& Stabler, M.J. (1997). Tourism economics, London: Routledge.

SUZUKI, J. (2013). Land use regulation as a barrier to entry: evidence from the Texas lodging industry. International Economic Review, 54 (2), 495-593. https://doi.org/10.1111/iere.12004

Tsang, E.W.K. \& Yip, P.S.L. (2009). Competition, agglomeration, and performance of Beijing hotels. Service Industries Journal, 29, 155-71. https://doi.org/10.1080/02642060802294896 
Tung, G.S.; LIN, C.Y. \& Wang C.Y. (2010). The market structure, conduct and performance paradigm re-alied to the international tourist hotel industry. African Journal of Business Management, 4(6), 1116-1125.

Vivel-Búa, M., Lado-Sestayo, R., \& Otero-González, L. (2016). Impact of location on the probability of default in the Spanish lodging industry A study of MSMEs. Tourism Economics, 22(3), 593-607. https://doi.org/10.5367/ te. 2015.0461

Vivel-Búa, M.; Lado-Sestayo, R. \& Otero-González,L. (2018). Risk determinants in the hotel sector: Risk credit in MSMEs. International Journal of Hospitality Management, 70, 110-119. https://doi.org/10.1016/j.ijhm.2017.11.004

Vives, A., Jacob, M., \& Payeras, M. (2018). Revenue management and price optimization techniques in the hotel sector: A critical literature review. Tourism Economics, https://doi.org/10.1177/1354816618777590

Williamson, O. E. (1975). Markets and hierarchies. New York, 2630.

Williamson, O. E. (1985). The economic institutions of capitalism: Firms, markets, relational contracting. London: Macmillan

Yang, Y., Tang, J., Luo, H., \& Law, R. (2015). Hotel location evaluation: a combination of machine learning tools and web GIS. International Journal of Hospitality Management, 47, 14-24. https://doi.org/10.1016/j.ijhm.2015.02.008

Yeap, C.A. (2011). Capacity use in multi-unit firms: evidence for efficiency gains or strategic competition in the US restaurant industry? Applied Economics, 43 (22), 2953-2968. https://doi.org/10.1080/00036840903299797

Yeung, P. K., \& Lau, C. M. (2005). Competitive actions and firm performance of hotels in Hong Kong. International Journal of Hospitality Management, 24(4), 611-633. https://doi.org/10.1016/j.ijhm.2005.06.005

Zhao, J. L. (1994). The antecedent factors and entry mode choice of multinational lodging firms: the case of growth strategies into new international markets. Tesis Doctoral. Faculty of Virginia Polytechnic Institute and the State University, United States. 